\title{
Stress Analysis of a Tooth Restored with a Post and Core
}

\author{
M.C.R.B. PETERS, ${ }^{*}$ H.W. POORT, ${ }^{+}$J.W. FARAH, ${ }^{\dagger}$ and R.G. CRAIG ${ }^{\dagger}$
}

* Department of Cariology and Endodontology, University of Nijmegen, P.O. Box 9101,6500 HB Nijmegen, The Netherlands; ${ }^{+}$School of
Dentistry, Academic Hospital V.U.B., Free University of Brussels, Belgium; and $†$ Department of Dental Materials, School of Dentistry, Uni-
versity of Michigan, Ann Arbor, Michigan 48109

An idealized axisymmetric finite element model of a second premolar restored with a post and core was used to study the distribution and magnitude of stresses as a function of the following parameters: the diameter of the post, the length and the shape of the post, and finally the interface characteristics between post and cement. Emphasis was directed toward the cement layer interposed between the post and the tooth. Bonding between the post and the cement appeared to be the most important parameter to achieve optimal mechanical behavior of the tooth-prosthesis combination.

J Dent Res 62(6):760-763, June 1983

\section{Introduction.}

A cast gold post with core has long been advocated for rehabilitation of endodontically treated teeth. However, such a restoration may result in complications such as loosening of the post and core or fracture of the roots. The importance of adaptation of the interfaces on the stresses in restored teeth has been shown in former research. 1

Clinical longevity of the post-and-core restoration can be a function of the thickness of the remaining dentin, the magnitude and direction of the load, the design of the post, the fit of the post into the prepared root canal, and the quality of the cement layer. Loading of a cemented post and core in a root creates stresses in the root, and if the tensile stresses are high, fracture of the root may occur. Frequent loading may cause strains and stresses in the cement layer that could result in damage of the cement film and consequent release of the post. Research has been reported on the effect of various types of posts on the stresses in the dentin and the bone. ${ }^{2,3}$ The magnitude and distribution of the stresses occurring in the cement have received little, if any, attention.

The aim of this investigation is to analyze the stress distribution in a root with a post-and-core restoration. The investigation is directed mainly at the cement film between the post and the dentin, with special emphasis on the postcement interface.

\section{Materials and methods.}

A well-known procedure for calculating stresses in a complex structure is the finite element method. ${ }^{4,5}$ This method, a computerized mathematical technique, was used to study the stresses in an idealized, endodontically treated maxillary second pre-molar. The finite element method allows one to simulate the loading as well as the various elastic properties of the restored tooth. To obtain the stresses and displacements throughout the model, the elastic constants - E (modulus of elasticity) and $\nu$ (Poisson's ratio) - must be known. These constants were obtained from the literature (Table) and were assigned to the appropriate regions. Two types of loading conditions were

Received for publication July 14, 1982

Accepted for publication August 24, 1982

This research was supported by a Fulbright Grant from ICA under PL \#87-256. simulated on the above model: (a) concentrated (X1), and (b) distributed (X2), as shown in Fig. 1 .

In the first part of this investigation, three post design parameters were evaluated: the diameter, the length, and the shape. In this case, the post-cement interface was assumed to be perfectly bonded. The various designs are shown in Fig. 1.

In the second part of the investigation, the post-cement interface was assumed to be either perfectly bonded or friction-held, in order to determine the influence of bonding on the stress distribution. The friction coefficient used was $\mu=0.1$. In this case, three post designs were examined: (a) long cylindrical design (Fig. 1B); (b) short cylindrical design (Fig. 1E); and (c) tapered design (Fig. $1 \mathrm{G})$.

\section{Results.}

The stresses examined were the cquivalent stresses, which give an indication of the sensitivity of the model to the applied load. An example of an equivalent stress plot is shown in Fig. 2. The stress lines in this drawing connect points with identical levels of stress. The plot can be read as a geographical map with relief lines.

Axial stresses $\left(\sigma_{z}\right)$ resulting from concentrated loading (X1) were evaluated in the cement along the line PQ (Fig. 1). The effect of diameter variation in the long cylindrical designs (A vs. B vs. C) on the axial stresses in the cement layer is shown in Fig. 3. The axial stresses were uniformly distributed, and the small diameter design (C) experienced the highest stress values. In Fig. 4, the axial stresses are shown for the shape variations (A vs. F vs. G), evaluating the influence of cylindrical $v s$. tapered designs. Although near the core (P) minimal differences occurred between the compared designs, more significant differences were observed toward the apex of the roots. The tapered post $(\mathrm{G})$ resulted in stress values of $10 \mathrm{MPa}$ as compared to $8 \mathrm{MPa}$ in designs $\mathrm{F}$ or $\mathrm{A}$. Fig. 5 shows the axial stresses when variation in length was considered ( $A$ vs. D vs. E). We should point out that the peak stresses in designs $E$ and $D$ are attained near the tip of the post. It is evident from Fig. 5 that, in general, the axial stresses were more uniformly distributed for design A.

Examination of the calculated stresses for a distributed load (X2) resulted in comparable stress patterns throughout the cement region.

TABLE

ELASTIC CONSTANTS FOR MATERIALS

\begin{tabular}{lcc}
\hline Material & E(MPa) & $\nu$ \\
\hline Bone (7) & $1.38 \times 10^{4}$ & 0.30 \\
PDL (7) & 3.45 & 0.45 \\
Dentin (7) & $1.86 \times 10^{4}$ & 0.31 \\
Cement (6, 8) & $1.37 \times 10^{4}$ & 0.35 \\
Post \& Core (9) & $7.72 \times 10^{4}$ & 0.33 \\
Gutta Percha (10) & 0.69 & 0.45 \\
\hline
\end{tabular}

\#umbers in parentheses denote references. 


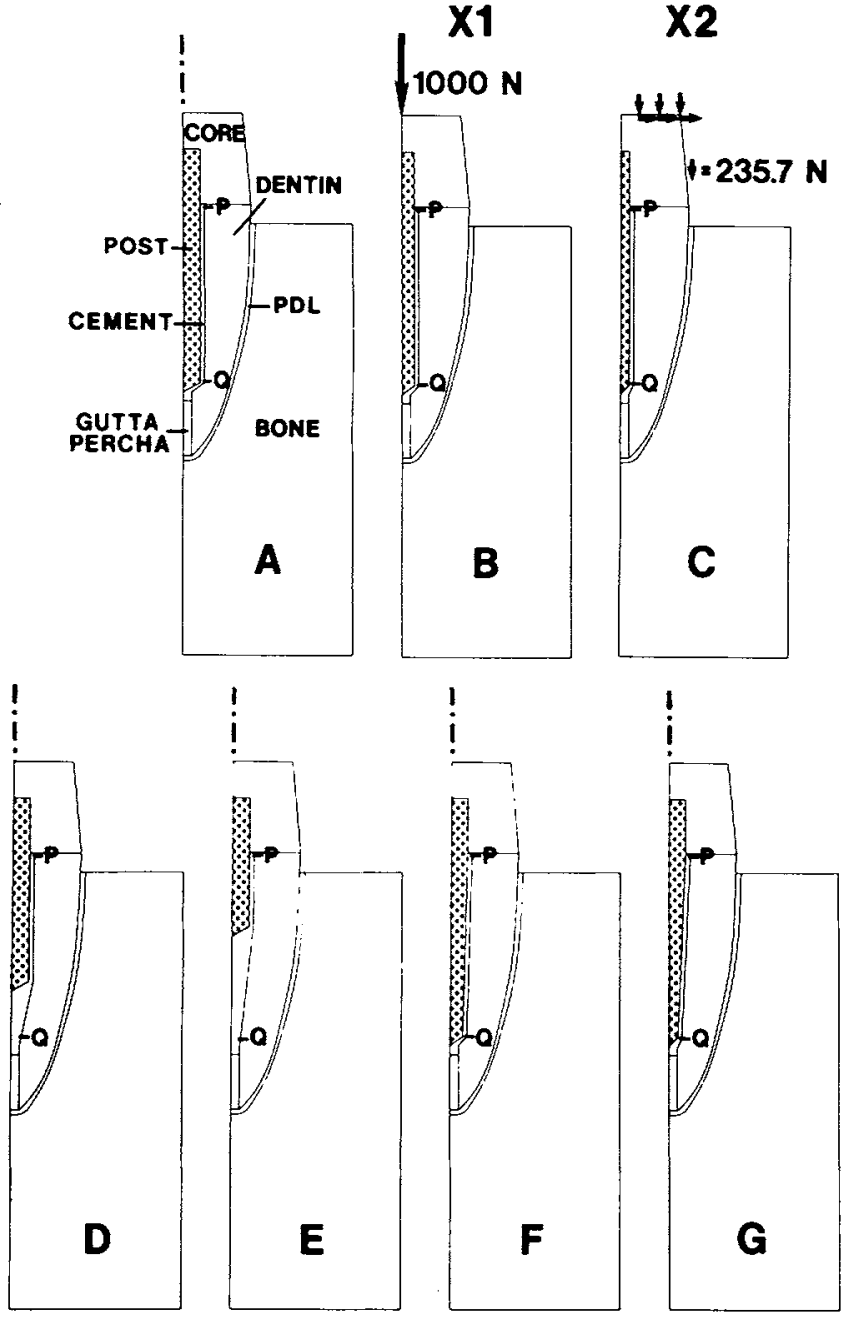

Fig. 1 - Seven different post designs. Three long cylinders (length, $10.5 \mathrm{~mm}$ ) with various diameters (A: $2.0 \mathrm{~mm} ; \mathrm{B}: 1.5 \mathrm{~mm}$; and $C: 1.0 \mathrm{~mm})$ and two cylinders $(\emptyset: 2.0 \mathrm{~mm})$ with various lengths: medium (D:7.5 mm) and short (E:4.5 mm). Two tapered post designs (length, $10.5 \mathrm{~mm}$; cervical, $\emptyset: 2.0 \mathrm{~mm}$ ) with various apical diameters (F:1.5 mm and $G: 1.0 \mathrm{~mm}$ ). Loading conditions $\mathrm{X} 1$ and $\mathrm{X} 2$ as well as PQ (abscissa in Figs. 3-8) are shown.

For evaluation of the effect of bonding between post and cement, three designs were chosen: B, E, and G. The axial stresses in the cement layer were examined at loading condition X1 (see Figs. 6-8). In model B (Fig. 6), the overall level of stress in the case of friction was about $20 \%$ higher when compared to the connected interface. The axial stresses in the case of friction increased considerably toward the apex, whereas the connected boundary resulted in a uniform distribution of stresses throughout the cement layer. Considering the tapered post (Fig. 7), the friction interface also resulted in higher stress values. Compared to the connected interface, the axial stresses near the core (P) appeared to be about $40 \%$ higher. When the short post design was considered (Fig. 8), the axial stresses were substantially affected by the boundary conditions chosen. In the cement region surrounding the post, the friction boundary resulted in at least $50 \%$ higher stress values in comparison with the connected boundary. Peak stresses (13 MPa) were developed in the vicinity of the tip of the post. On the contrary, the connected boundary resulted in peak stresses (14 MPa) located just apical to the tip of the post.

A distributed load (X2) applied to the core resulted in

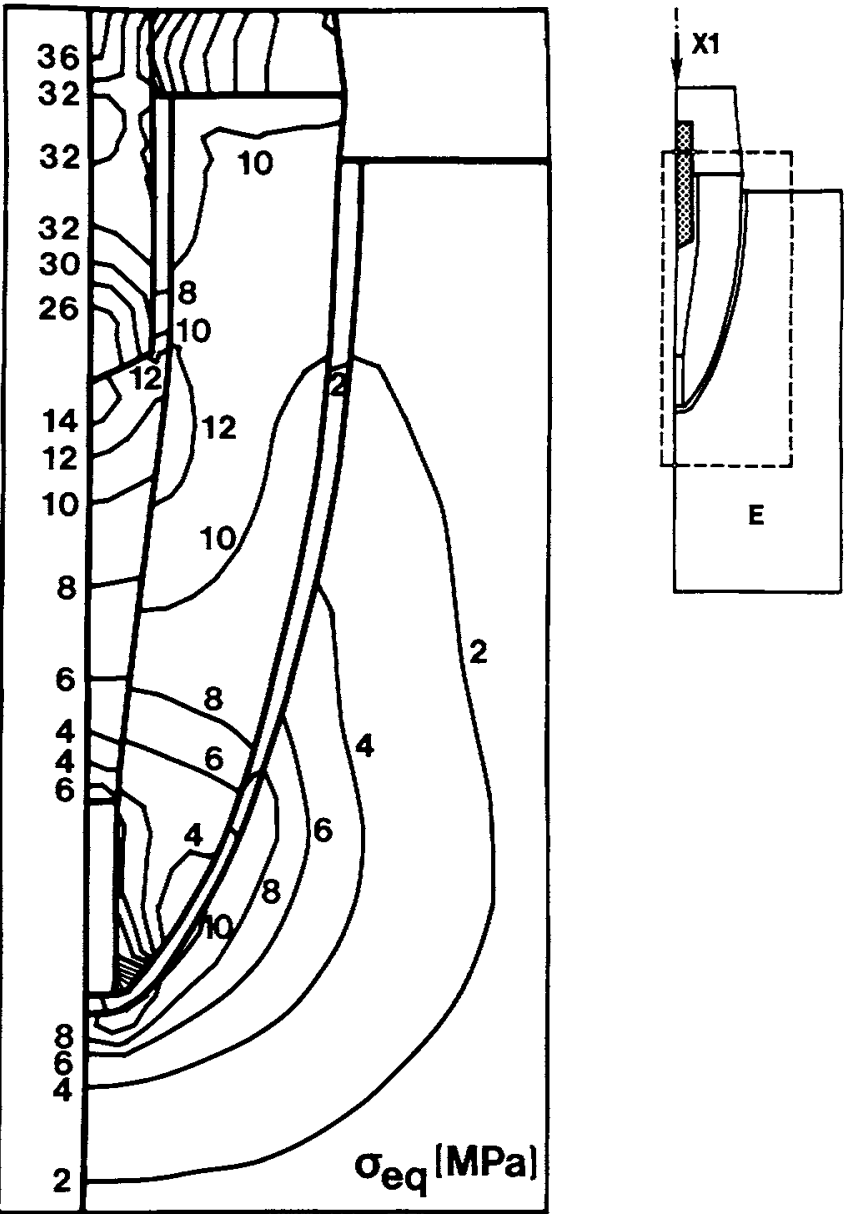

Fig. 2 - Levels of equivalent stress within the indicated area of model E. A concentrated load (X1) was applied, and the postcement interface was assumed to be perfectly bonded.

comparable yet less distinct stress distributions in the cement layer. The induced stress patterns showed the same tendencies. A typical plot of axial stresses (Fig. 9) shows the stress concentrations in the cement in the vicinity of the tip of the short post. At loading condition X2, the axial stresses are plotted throughout the model with friction at the interface. Even under distributed loading, the cement

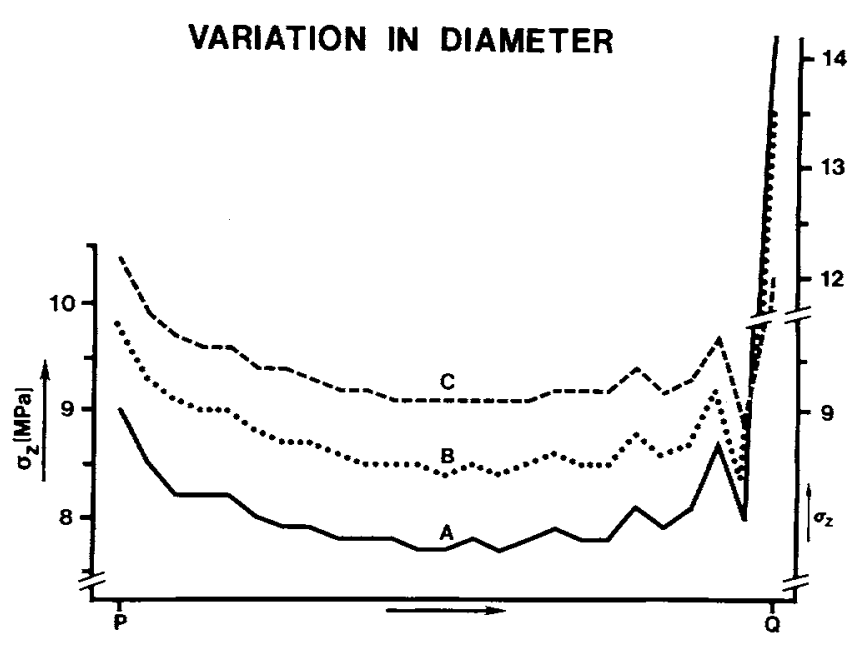

Fig. 3 - Graphic presentation of the axial stresses in the cement layer of models $\mathrm{A}, \mathrm{B}$, and $\mathrm{C}$ at concentrated loading. 


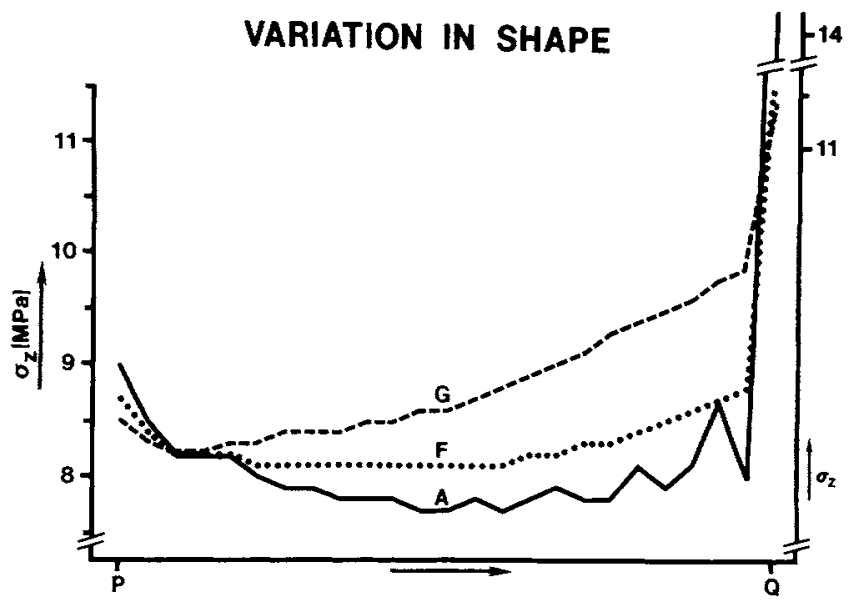

Fig. 4 - Graphic presentation of the axial stresses in the cement layer of models $A, F$, and $G$ at concentrated loading.

VARIATION IN LENGTH

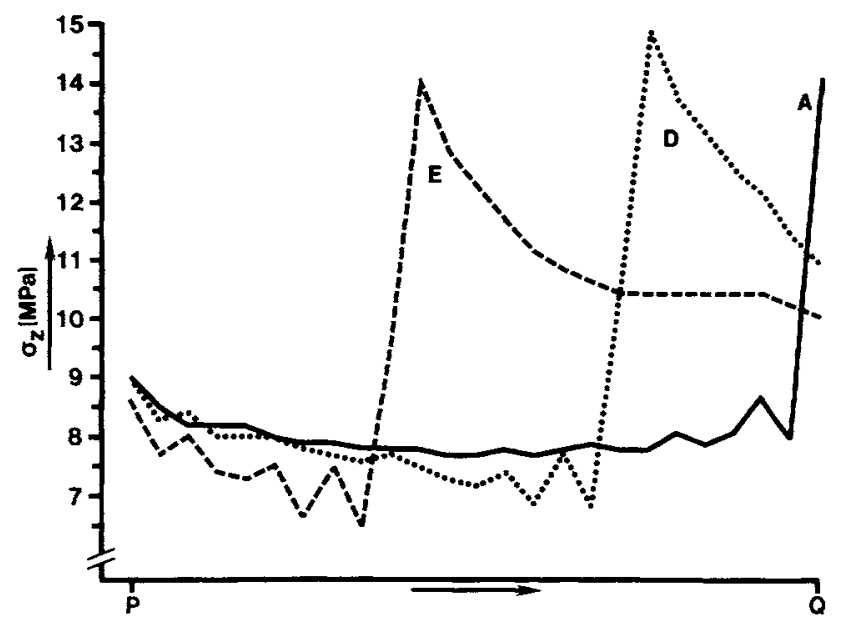

Fig. 5 - Graphic presentation of the axial stresses in the cement layer of models $\mathrm{A}, \mathrm{D}$, and $\mathrm{E}$ at concentrated loading.

experiences considerable stress gradients in contrast with the post.

\section{Discussion.}

In a clinical situation, the load is applied to the crown which, in turn, transfers such a load to the core. The crown will therefore distribute the forces evenly to the core. In the present study, the load was applied directly to the core, but it was found that a more distributed type of loading resulted in identical stress patterns. Although a rather high loading force was used, the magnitude of the applied loads can easily be reached when bruxism is involved. Two tensile strength values - reported in the literature ${ }^{6}$ for zinc phosphate cement as the luting agent - are 4.3 and 8.1 $\mathrm{MPa}$. Considering the axial stresses computed in this study, these strength values of zinc phosphate cement can be exceeded. Although no real failure criterion is known, one could deduce that under certain conditions, when the above tensile stresses are exceeded, failure to the cement can occur.

The various post geometries, examined in this study under axisymmetric loading, resulted in minor differences in stress distribution throughout the remaining tooth structures and the supporting bone. Within the dentin, the

\section{LONG CYLINDER B}

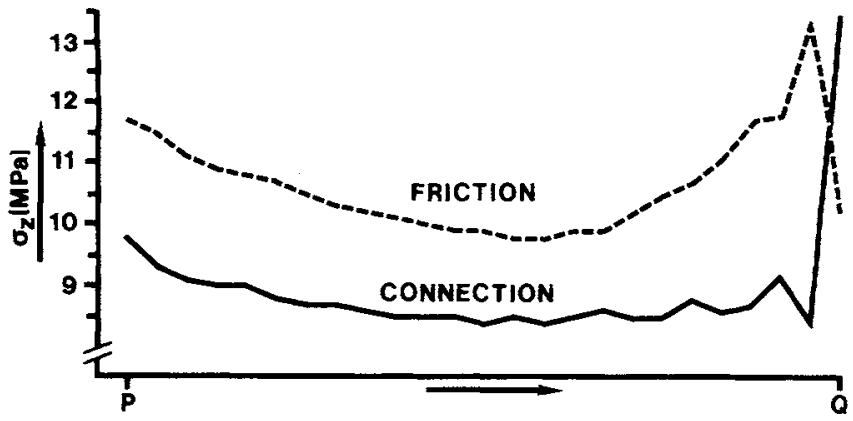

Fig. 6 - Graphic presentation of the axial stresses in the cement layer of model $B$ at concentrated loading with different boundary conditions.

TAPER $\mathbf{G}$

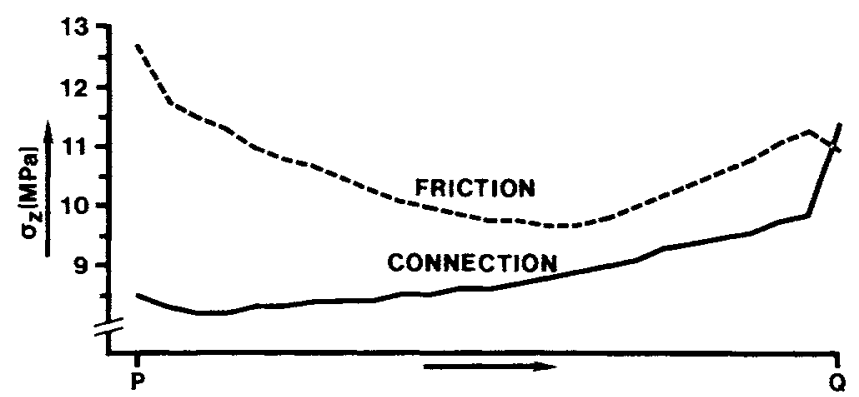

Fig. 7 - Graphic presentation of the axial stresses in the cement layer of model $G$ at concentrated loading with different boundary conditions.

\section{SHORT CYLINDER E}

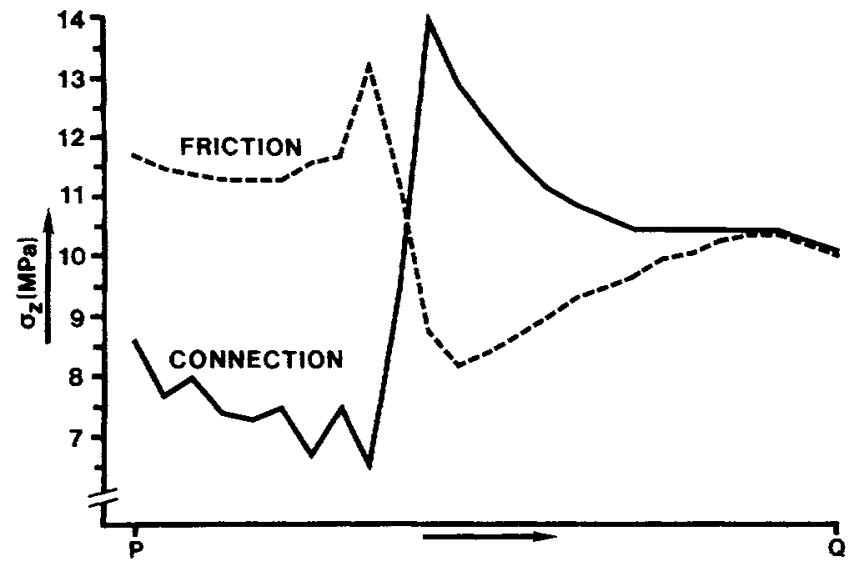

Fig. 8 - Graphic presentation of the axial stresses in the cement layer of model $E$ at concentrated loading with different boundary conditions.

short cylindrical design had the highest values for the equivalent stresses (Fig. 2). The cement layer exhibited more significant differences in the stresses. If lateral as well as axisymmetric forces are applied, one would then expect the stresses within the cement, dentin, and bone to increase more dramatically. This would be true because lateral forces would result in a bending force on the core and post. 

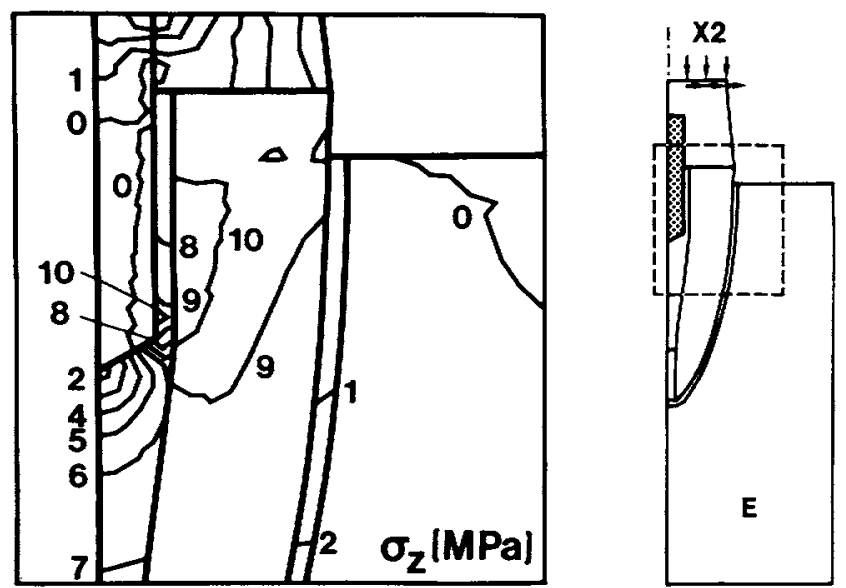

Fig. 9 - Levels of axial stress within the indicated area of model E. A distributed load (X2) was applied, and the post-cement interface was assumed to be friction-fitted.

The high peak stresses at point $Q$ are caused not only by the various parameters but also by the modeling of the post and the dentin. The apical angulation of the post and the geometry of the root canal strongly influence the stresses at point $Q$. Therefore, they will not be discussed in detail.

When a perfect bond was assumed between the post and the cement as well as the dentin, similar stress patterns were obtained for each diameter of the cylindrical post (Fig. 3). As the diameter of the post was doubled (design A vs. design $\mathrm{C}$ ), the stress decreased by about $20 \%$. Obviously, one cannot increase the diameter of the post indefinitely, thereby increasing the likelihood of perforation of the root, or even the fracture of the root. An optimal ratio of post diameter to remaining dentin thickness can be calculated using the finite element method, but this was not within the scope of this investigation. Still, it is well to remember that the various post dimensions chosen for this study were within clinically acceptable limits.

A comparison of cylindrical vs. tapered designs (Fig. 4) showed that the effect of taper was most distinct in the apical region (near Q). The tapered design, G, when compared to the non-tapered $A$, caused an increase of about $25 \%$ in the axial stresses near the tip of the post. Minor changes in the overall stress pattern were caused by variation in the length of the post (Fig. 5). Variation in the length strongly influenced the location of the peak stresses in the cement. These peak stresses were higher than 14 $\mathrm{MPa}$; thus, they could contribute to failure of the cement.

As a result of the modeling of the core-dentin interface and the applied loads, the dentin uniformly supported the core. However, even with such support, the results showed noticeably higher stress values in the cement when the bonding conditions were changed from that of a perfect bond to a friction-fit bond. In Figs. 6, 7, and 8, both interface conditions were compared in three different geome- tries of the post. It is evident that the modeling of the interface is the most important parameter. In the frictiontype boundary, the stresses within the post were almost negligible when compared to the stresses within the cement, which were about $10 \mathrm{MPa}$ at loading X1 (Figs. 6-8). Even in the case of loading X2, the axial stresses in the cement could reach a value of $10 \mathrm{MPa}$ (Fig. 9). Such a large stress gradient between two surfaces is detrimental and could be the cause for further failure of the postcement interface.

\section{Conclusions.}

Using an idealized axisymmetric model and the loading conditions considered, it was found that, in the case of perfect bonding, a slight preference could be given to a long cylindrical post with a large diameter if clinical limits were not exceeded. High peak stresses appeared in the cement layer between the post and the dentin when the bond between their surfaces was imperfect.

It was found that the tensile strength value of zinc phosphate cement could easily be exceeded. This mathematical study put emphasis on the importance of achieving optimal bonding when cementing a post-and-core restoration.

\section{REFERENCES}

1. PETERS, M.C.R.B. and POORT, H.W.: Biomechanical Stress Analysis of the Amalgam-Tooth Interface, $J$ Dent Res, In press.

2. STANDLEE, J.P.; CAPUTO, A.A.; COLLARD, E.W.; and POLLACK, M.H.: Analysis of Stress Distribution by Endodontic Posts, Oral Surg 33:952-960, 1972.

3. DAVY, D.T.; DILLEY, G.L.; and KREJCI, R.F.: Determination of Stress Patterns in Root-filled Teeth Incorporating Various Dowel Designs, J Dent Res 60:1301-1310, 1981.

4. CRAIG, R.G. and FARAH, J.W.: Stress Analysis and Design of Single Restorations and Fixed Bridges, Oral Sci Rev 40:45-74, 1977.

5. PETERS, M.C.R.B.: Biomechanics of Cavity Preparation and Restoration of Human Teeth; Modeling and Analysis with the Finite Element Method. Ph.D. dissertation, University of Nijmegen, The Netherlands, 1981, p. 304.

6. POWERS, J.M.; FARAH, J.W.; and CRAIG, R.G.: Modulus of Elasticity and Strength Properties of Dental Cements, JADA 92:588-591, 1976.

7. CRAIG, R.G. and FARAH, J.W.: Stresses from Loading Distalextension Removable Partial Dentures, $J$ Prosthet Dent 39: 274-277, 1978.

8. HALL, D.R.; NAKAYAMA, W.T.; GRENOBLE, D.E.; and KATZ, J.L.: Elastic Constants of Three Representative Dental Cements, $J$ Dent Res 52:390, 1973.

9. CRAIG, R.G.; PEYTON, F.A.; and JOHNSON, D.W.: Compressive Properties of Enamel, Dental Cements and Gold, $J$ Dent Res 40:936-945, 1961.

10. GREENER, E.H.; HARCOURT, J.K.; and LAUTENSCHLAGER, E.P.: Material Science in Dentistry, Baltimore, MD: Williams and Wilkins, 1974, p. 408. 